\title{
A NOVEL CRYOGENIC LIQUID LEVEL SENSING TECHNIQUE
}

\author{
Pragyaditya Das ${ }^{1}$ \\ ${ }^{I}$ Department of Instrumentation and Control Engineering, National Institute of Technology, Trichy, Tamilnadu \\ 620015, India
}

\begin{abstract}
This paper explores the use of a light based measurement technique for the level measurement of cryogenic liquids. Differential pressure transmitters are commonly used to measure the levels of cryogenic liquids such as liquid nitrogen and liquid oxygen in cryogenic tanks. They require the liquid inside the temperature controlled tank to be drawn out into impulse lines which are at ambient temperature. This causes a number of problems, choking of the impulse pipes is one of them, which ultimately results in a need for flushing the lines and that causes loss of material.
\end{abstract}

Keywords: Cryogenics; Optical Level Measurement

\section{INTRODUCTION}

Cryogenic liquids are of huge importance in today's fast growing Industrial Scenario. Storing them and undertaking tasks like level measurement, flow control etc. on them is another Herculean task to do. Due to their unusually low temperature, these liquids have some special problems to be dealt with.

\section{PROBLEMS FACED IN LEVEL MEASUREMENT OF CRYOGENIC LIQUID}

In this section, I shall put light on the problems faced while conducting the level measurement of a cryogenic liquid. I shall take the case of Liquid Nitrogen (LIN). Now, just for information, I shall mention some salient features of LIN,

Table 1: Details of Liquid Nitrogen

\begin{tabular}{|c|c|}
\hline Property & Value \\
\hline Temperature (in ${ }^{\circ} \mathrm{C}$ ) & $-196^{\circ} \mathrm{C}$ \\
\hline Temperature (in ${ }^{\circ} \mathrm{F}$ ) & $-321^{\circ} \mathrm{C}$ \\
\hline Refractive Index & $1.1990^{[1]}$ \\
\hline
\end{tabular}

Needless to say that, when we are aiming at the use of Differential-pressure (DP) method for the measurement of level of liquid nitrogen, we will have to make two tapping on the dewar chamber for impulse lines; one at the High Pressure area and one at the Low Pressure area. Let us say, we successfully install the DP level transmitter, now when the LIN comes into the impulse tubes, the catastrophe starts. As data suggests ${ }^{[2]}$,Carbon Di-oxide becomes dry ice at $78.5^{\circ} \mathrm{C}$, so the LIN coming into the impulse pipe comes in contact with the $\mathrm{CO}_{2}$ in the pipe and immediately turns the $\mathrm{CO}_{2}$ into dry ice. This leads to the choking of the impulse pipe.

A table of the level readings taken at an interval of 12 hours, in no-delivery-condition is as follows,
Table 2: Level using DP Method - No Delivery mode

\begin{tabular}{||cc||}
\hline Time(Hours) & Level(mtrs.) \\
\hline \hline 2 & 171.5 \\
\hline 5 & 150.3 \\
\hline 7 & 140.9 \\
\hline 10 & 138.1 \\
\hline 12 & 136.3 \\
\hline
\end{tabular}

We can see that the level seems to continuously go down due to the effect of chocking and not due to delivery.

\section{STUDY OF LIN}

Liquid Nitrogen exists at extremely low temperatures and hence is of great interest to Cryogenic physicists and Engineers. There are a lot of salient points that are ought to be known while dealing with LIN. I will take the help of bullets to put forth the details in a more comprehensible manner. The details that I am going to present are from various online sources ${ }^{[3],[4]}$, some salient features are as follows,

1) Liquid Nitrogen is denoted as LN, LIN or LN2.

2) The UN number given to LIN is 1977.

3) At NTP, LIN boils at $-195^{\circ} \mathrm{C}$

4) It is non-toxic and inert. Hence, finds a lot of industrial applications for inert blanketing of Petrochemical products.

5) LN2 displays the Leidenfrost effect ${ }^{[5]}$.

\section{PROPOSED DESIGN}

In this section, I will put forward my proposed design to tackle the problems mentioned in above sections. To put forth in a very simple manner, I will be taking the aid of optical properties of LIN and air to design a novel optical level measurement mechanism for cryogenic liquid. The reason why any kind of wave based sensor, say, Ultrasonic or RADAR sensors will fail, is because that will require an open vessel. And, as in the case of Cryogenic Liquids, open vessel is not an option. 
A simple set-up is proposed that takes care of the very basic drawback using the optical properties of cryogenic liquid can be seen in Fig. 1.

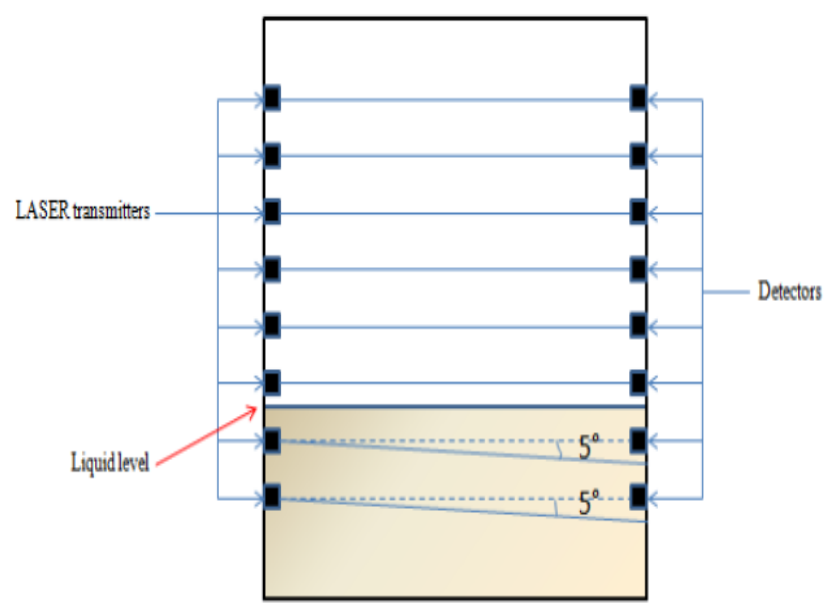

Fig 1: Setup

For design purpose I used LASER light source, however, my observation is, the use of any light source is applicable with enough monochromatic nature. The design is heavily dependent on the basis of Snell's Law in Refraction mechanism. The design is heavily analyzed mathematically. The next section will provide the detail ideation of the mathematical background of the design.

\section{ANALYSIS (MATHEMATICS BEHIND THE}

\section{IDEA)}

At the very onset, let me first define all the notations for easier reference throughout the length of the paper,

We have,

1) $\rho=$ Density $\left(\mathrm{kg}=\mathrm{m}^{3}\right)$

2) $\mathrm{m}=$ Mass $(\mathrm{kg})$

3) $\mathrm{V}=$ Volume $\left(\mathrm{m}^{3}\right)$

4) $A=\operatorname{Area}\left(\mathrm{m}^{2}\right)$

5) $\mathrm{F}=$ Force $(\mathrm{N})$

6) $g=$ acceleration due to gravity $\left(9: 81 \mathrm{~m}=\mathrm{s}^{2}\right)$

7) $1=$ Length $(\mathrm{m})$

8) $\mathrm{P}=$ Pressure $\left(\mathrm{N}=\mathrm{m}^{2}\right)$

9) $\mu=$ Refractive Index.

10) $\mathrm{T}=$ Temperature $\left({ }^{0} \mathrm{C}\right)$

11) $\lambda=$ Wavelength $(\mathrm{m})$

12) $\mathrm{k}=$ Wavenumber $\left(\mathrm{m}^{-1} 1\right)$

We will eastablish some basic rules on which we will build our mathematics at the later stages,

We know that,

$$
\rho=\frac{m}{V}
$$

Which can be written as,

$$
\rho=\frac{m}{l} \times \frac{1}{A}
$$

In equation (2) we can have ( $\mathrm{m}$ ) of the material and (A) covered by the material to be the varying quantities, but the (1) of the container remains constant. Therefore, we can write,

$$
\rho \propto \frac{m}{A}
$$

Similarly, we can now have a look at the idea of Pressure,

$$
P=\frac{F}{A}
$$

which can be written as,

$$
P=\frac{m}{A} \times g
$$

It is obvious that $(\mathrm{g})$ remains constant in normal conditions,

Therefore, we can conclude that,

$$
P \propto \frac{m}{A}
$$

From (3) and (4) we conclude that,

$$
\rho \propto P
$$

Keeping that in mind, we must also keep in mind that the density is synonymous to Particle/Matter density.

Now, as per [6], to define the relation between the refractive index of air with respect to temperature, we have,

$$
\mu=1-\sum n_{i} \delta_{i}(k)
$$

Note that this is valid for small wave numbers(k), which in one way means, the above equation is valid for longer wavelengths, since, $\mathrm{k}=\lambda^{-} 1$. 
In equation (8), we must note that, $\eta_{i}$ is the number density for each species of molecule and $\delta_{\mathrm{i}}$ is the contribution to the index from this molecular species.

Clearly, if we increase the pressure $(P)$, the density $(\rho)$ also increases, as per (7). Thus, when ( $\rho$ ) increases, $\mu$ also increases. Similarly, $\mu$ and $\mathrm{T}$ are inversely related.

Therefore, mathematically we can write,

$$
\mu=\frac{P}{T}
$$

Another interesting point of view into this idea is by taking into account the Sellmeier Equation, which defines the empirical relationship between the wavelength and refractive index for a particular transparent medium. This relation closely follows the $\mathrm{y}=\mathrm{e}^{-\mathrm{mXx}}$, where $\mathrm{m}$ is a positive integer. Where, y is Refractive Index and $x$ is Wavelength. Similarly, we know it well the fact that, hot objects emit more of their light at short wavelengths, and cold objects emit more of their light at long wavelengths. The temperature of an object is related to the wavelength at which the object gives out the most light. [7]

Now applying a little logic solves the problem. For low temperature, the $\lambda$ becomes long and hence, $\mathrm{k}$ becomes small. Therefore, $\mu$ goes up. Keeping in mind that $\mathrm{P}$ is isolated. Or, in other words, the system is Isobaric.

Similarly, in an Isothermal system, $P$ and $\mu$ are directly related.

Lastly, the very basis of the setup's working is based on the phenomenon of bending of light when it runs from a rarer to a denser medium. So, just for completeness I will add Snell's law,

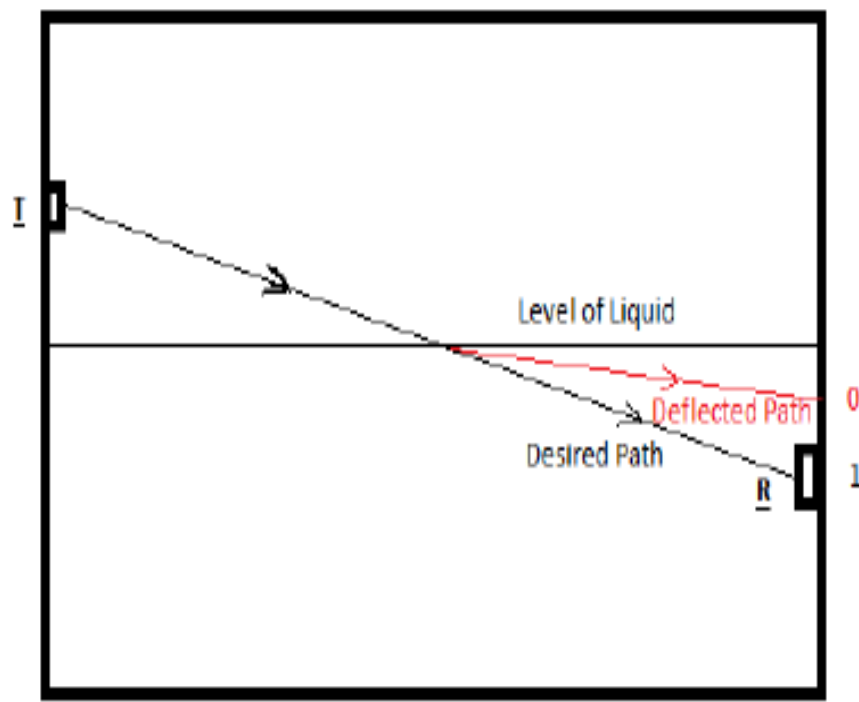

Fig. 2: Design

$$
\frac{\mu_{N_{2}}}{\mu_{\text {air }}}=\frac{\sin \theta_{\text {air }}}{\sin \theta_{N_{2}}}
$$

Adding some values into (10). We know that, $\mu_{\text {air }}=1$ and from [1], it is found that $\mu_{\mathrm{N} 2}=1.2$.

Therefore,

$$
\frac{\sin \theta_{\text {air }}}{\sin \theta_{N_{2}}}=\frac{1.2}{1}
$$

Having the $\sin \Theta_{\text {air }}=10^{\circ}$, we can find

$$
\begin{gathered}
\sin \theta_{N_{2}}=\sin \theta_{\text {air }} \times \frac{1}{1.2} \\
\sin \theta_{N_{2}}=10^{\circ} \times \frac{1}{1.2}
\end{gathered}
$$

We have,

$$
\sin \theta_{N_{2}}=8.333^{\circ}
$$

The design method is pictorially depicted in fig. 2 .

\section{DESIGN}

The black line shows the path of the light beam when there is no liquid obstructing its path. In that case, the output at the receiver is 1 . Similarly, the red line shows the path of the light beam when the liquid obstructs its path. In that case, in accordance to Snell's law, the beam deviated and missed the receiver it was initially aimed at, therefore, the output becomes 0 . Hence, giving an indication that the level has surpassed that particular receiver(R).

We must note that, the accuracy of the design depends heavily on the number of Transmitters and Receivers used. The more the density of the Transmitters and Receivers, the more will be the accuracy.

\section{RESULTS}

The results are tabulated for clarity. The following table gives the level of a dewar container in no-delivery mode.

Table 3: Level using Optical Method - No Delivery mode Time (Hours) Level(mtrs.)

\begin{tabular}{||cc||}
\hline Time(Hours) & Level(mtrs.) \\
\hline \hline 2 & 180 \\
\hline 5 & 179.8 \\
\hline 7 & 179.8 \\
\hline 10 & 179.7 \\
\hline 12 & 179 \\
\hline
\end{tabular}


The data clearly shows that the method significantly improves the performance.

\section{CONCLUSION}

As we can see from Table 2 and Table 3 that there is a significant improvement in the performance of the system. There are some issues that come inevitable with such a system, some of them are; I suggest the use of a monochromatic light source for use in such application, I suggest the use of this system in a single-point critical system - a system in which either the high level OR low level are critical - not both.

\section{ACKNOWLEDGEMENT}

I would like to thank my teachers from NIT-Trichy, India; who guided me in understanding the basics of Instrumentation and Measurement Engineering. I would like to thank my parents and the almighty God for their blessings and support.

\section{BIOGRAPHY}

Pragyaditya Das graduated from National Institute of Technology, Trichy, India. Being from one of the premeire institutes of India, he got exposed to a whole host of great people to interact with. He is interested in Sensor System Design, Smart Instrumentation and Advanced Control Systems.

\section{REFERENCES}

[1] H. E. Johns and J. O. Wilhelm, The Refractive Indices Of Liquid Oxygen, Nitrogen, And Hydrogen, Volume 15. Canadian Journal of Research, July 1937.

[2] Wikipedia- Dry Ice,

[3] Wikipedia- Liquid Nitrogen,

[4] https://www.thoughtco.com/liquid-nitrogen-facts608504- Liquid Nitrogen Facts,

[5] Wikipedia - Leidenfrost effect,

[6] https://physics.stackexchange.com/questions/6872/ref ractive-index-ofair-in-dependence-oftemperature/1494814948 - Refractive index of air in dependence of temperature,

[7] http://hubblesite.org/referencedesk=faq=answer:php:i $\mathrm{d}=74$ cat =light $\square$ How are wavelength and temperature related?; 